\title{
Potential Applications of Active Antenna Technologies for Emerging NASA Space Communications Scenarios
}

\author{
James A. Nessel ${ }^{1}$, James M. Downey ${ }^{1}$, Bryan L. Schoenholz ${ }^{1}$, Marie T. Piasecki ${ }^{1}$, Félix A. Miranda ${ }^{1}$ \\ NASA Glenn Research Center: Communications and Intelligent Systems, Cleveland, OH, USA, james.a.nessel@nasa.gov
}

\begin{abstract}
The National Aeronautics and Space Administration (NASA) is presently embarking on the implementation of far-reaching changes within the framework of both space and aeronautics communications architectures. For example, near earth relays are looking to transition from the traditional few large geostationary satellites to satellite constellations consisting of thousands of small low earth orbiting satellites while lunar space communications will require the need to relay data from many assets distributed on the lunar surface back to earth. Furthermore, within the aeronautics realm, satellite communications for beyond line of sight (BLOS) links are being investigated in tandem with the proliferation of unmanned aerial systems (UAS) within the urban air mobility (UAM) environment. In all of these scenarios, future communications architectures will demand the need to connect and quickly transition between many nodes for large data volume transport. As such, NASA Glenn Research Center (GRC) has been heavily investigating the development of low cost phased array technologies that can readily address these various scenario conditions. In particular, GRC is presently exploring 5G-based beamformer technologies to leverage commercial timescale and volume production cycles which have heretofore not existed within the frequency allocations utilized for NASA applications. In this paper, an overview of the potential future applications of phased arrays being envisioned by NASA are discussed, along with technology feasibility demonstrations being conducted by GRC implementing low cost, 5G based beamformer technologies.
\end{abstract}

Index Terms-active phased array antennas, 5G technology, small satellites, space communications, aeronautics communications.

\section{INTRODUCTION}

The communications ecosystem for space and aeronautics is undergoing radical changes over the next decade and the need for phased array technologies is becoming increasingly apparent to meet future demands and system requirements. Within these envisioned next generation aerospace communications architectures, the primary driving factors include the proliferation of the number of nodes (i.e., assets) requiring connectivity, both on the user side and the relay side, and the increased use of shared spectrum which imposes non-interference requirements for all communicating assets operating in these bands. Furthermore, future platforms with communications needs continue to increase in quantity and reduce in size (e.g., CubeSats/SmallSats and unmanned aerial vehicles (UAV)), demanding a low cost, low size, weight and power (SWaP) solution.

Based on these identified constraints, phased array technologies leveraging the burgeoning growth of the radiofrequency (RF) silicon industry presents a viable solution for future NASA needs for space-borne as well as aero platforms. On the user side, phased arrays have the flexibility to shape beams to generate nulls to reduce interference with other systems, as well as generate multiple beams and/or fast-steering beams to communicate with multiple assets. This approach also provides a solution for handling multiple users on the relay side to maintain high data rates with minimal user burden imposed. Concomitantly, as industry continues to develop more efficient, higher performance silicon-based RF Integrated Circuits (RFICs) in mass production, costs continue to decrease while capabilities continue to increase within smaller and smaller packages. Thus, it appears that the availability as well as the demand for low cost/low SWaP phased array component technologies has made the timing right for phased arrays to be a key player for next generation communications systems.

In this paper, we discuss current plans and activities being considered by NASA where phased array technologies are proving to be a critical component for future aerospace needs. Particularly, 5G-based technologies are emerging as a driving force for the development of phased array solutions for future NASA missions. Several demonstrations and concerns are also discussed in the context of 5G-based solutions for the envisioned next generation of aerospace communications architectures.

\section{THE BENEFITS OF 5G TECHNOLOGY}

There has been much fanfare on the promise that the $5^{\text {th }}$ generation (5G) of mobile broadband technologies will bring to the user community to increase the capacity of services. Within the terrestrial domain, the 5G architecture revolution taking place is shown depicted in Fig. 1. Fundamentally, this improvement is a result of the transition from broad coverage cells connecting many users (Fig. 1, left) to individual beams 
servicing single users (Fig. 1, right). By simply replacing lower gain antennas with high gain antennas on both the user and the base station sides, dramatic increases in capacity are possible. To realize this, 5G technologies will leverage wider bandwidths and advanced antenna technologies such as phased arrays for massive multiple-input multiple-output (MIMO) systems [2, 3]. This has led to the explosive growth in commercial radios and beamforming chipsets with higher efficiencies and operating frequencies than ever before $[4,5]$.

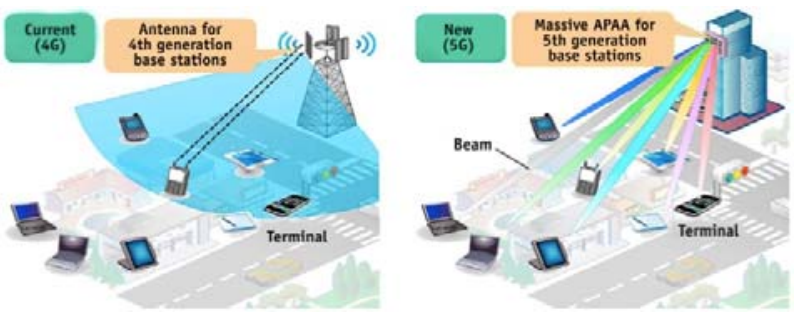

Fig. 1. Depiction of change in mobile network architecture from 4G (left) to $5 \mathrm{G}$ (right) systems indicating the transition from multiple users per cell to individual users per beam [1].

Of particular interest to NASA is that allocated spectrum in the n258 band (24.25-27.5 GHz) overlaps with the Kaband spectrum utilized for NASA space missions (25.5-27.5 $\mathrm{GHz}$ ), allowing the ability to leverage $5 \mathrm{G}$ technologies for NASA's space applications. Although concerns have been raised on the shared use of this spectrum (and rightly so), an opportunity has also arisen in that, for the first time, component/sub-system technologies needed for NASA communications systems has now become readily available, making what was once costly, custom designs of NASA's Ka-band systems much more affordable.

Presently, companies like Anokiwave, Analog Devices, Qualcomm, Integrated Device Technology (IDT), and many others, have been developing beamformer and downconverter chipsets that, if proven reliable in space, could support satellite communications (SATCOM) applications as well. It is envisioned that these technologies would remain low cost based on the tremendous volume of users and applications $5 \mathrm{G}$ is intended to support and may prove to be the tipping point for the proliferation of phased array technologies for both space and aeronautics applications.

However, it should be noted that there are differences within the 5G architecture that need to be worked with in order to make this approach more optimal for space architectures. For instance, 5G utilizes linear polarization and transmit and receive is accomplished over the same frequency band allocation. The latter imposes the use of a transmit/receive switch prior to the transmit/receive amplifiers, which results in increased front end loss (typically around $2 \mathrm{~dB}$ ) that reduces the output power of the system. For SATCOM, circular polarization is typically implemented and transmit and receive signals are operated in separate, distinct bands. As such, a linear to circular polarization conversion or phased dual linear operation needs to be implemented to meet space communication link requirements and a transmit/receive switch would not be implemented for SATCOM applications. However, it is feasible that simple design changes such as the removal of the receive path in the $5 \mathrm{G}$ chipset could be considered in future 5G beamformer chipsets optimized for space applications.

\section{PHASED ARRAYS FOR SATCOM APPLICATIONS}

\section{A. Near Earth Space Applications}

Within the context of near earth, the future space communications architecture is experiencing drastic changes. With the planned retirement of NASA's Tracking and Data Relay Satellite System (TDRSS) fleet in the 2040 timeframe, transitions to the use of commercial Ka-band services via satellite relays are being investigated to support future commercially-provided services for NASA missions. The use of commercial Fixed Satellite Service (FSS) Ka-band for inter-satellite links is presently forbidden, but steps are being taken to approve the use of this spectrum on a shared basis for space-to-space links [6]. Furthermore, next generation satellite communications architectures are presently being proposed by many commercial service providers (e.g., Space-X, Telesat, others) to include up to several thousand low earth orbiting relay satellites [7, 8]. In a future architecture such as this, the obvious need to switch across these many nodes necessitates the fast beam-steering capability of phased array technology.

Beyond space-based data relays, NASA missions are expected to continue to downlink data direct to earth, as well, and are planned to transition more fully into the Ka-band space research spectrum allocation (25.5-27.5 GHz). Many of the missions transitioning into this band are comprised of earth and planetary observation missions which typically utilize gimballed/fixed reflectors or iso-flux antennas (e.g., NASA-Indian Space Research Organization Synthetic Aperture Radar (NISAR), Plankton, Aerosol, Cloud ocean Ecosystem (PACE), Transiting Exoplanet Survey Satellite (TESS) missions). The former can cause issues with science instruments either due to vibrations induced through mechanical movement of the antenna while pointing to close the links, or the need to body-point the antennas, both of which interrupt science measurements. The latter eliminates this, but does so in an inefficient manner due to the generation of equal power flux density across the earth field of view. On the other hand, phased arrays, with their electrical steering and high directivity performance, have the capability to mitigate this problem. Furthermore, as science missions continue to exploit the use of small satellite architectures, the communications systems on these spacecraft become a dominating presence if high data volume return is needed. Within the CubeSat/SmallSat platform, phased array technology has yet to be introduced, but the development of low cost, low SWaP phased array technologies can now make this possible and transition the current state of practice body-pointing concepts of operations 
(CONOPS) in favor of non-body pointed solutions, potentially enabling new science capabilities.

Presently, NASA GRC is investigating the use of 5G technologies within the framework of CubeSats through the proposed flight demonstration of the SmallSat Ka-band Operations User Terminal (SKOUT). The SKOUT mission is looking to demonstrate 5G beamformer technology and software defined radios (SDRs) in space to test survivability and demonstrate the superior flexibility and functionality of phased arrays for CubeSats, as well as for future NASA missions. The specifications for the phased array being developed for the SKOUT mission are summarized in Table I.

TABLE I. PHASED ARRAY SPECIFICATIONS FOR THE SKOUT ARRAY

\begin{tabular}{|l|l|}
\hline Parameter & Final Specification \\
\hline Transmit Frequency Range & $24.25-29.5 \mathrm{GHz}$ \\
\hline Field of View & + - $60 \mathrm{deg}$ \\
\hline Polarization & RHCP \\
\hline Axial Ratio & $<3 \mathrm{~dB}$ over full FOV/frequency \\
\hline Instantaneous Bandwidth & $1 \mathrm{GHz}$ \\
\hline EIRP & $+30 \mathrm{dBW}$ (high power mode) \\
& $+20 \mathrm{dBW}$ (low power mode) \\
\hline Number of Tx beams & 1 \\
\hline DC Power Consumption & $<70 \mathrm{~W}$ (high power mode) \\
& $<30 \mathrm{~W}$ (low power mode) \\
\hline
\end{tabular}

SKOUT will operate across the n257 and n258 bands (24.25 - $29.5 \mathrm{GHz})$. In addition to the performance specification defined in Table I, individual element amplitude and phase control are available for full control of the output formed beam. Furthermore, additional communications payloads will accompany and integrate with the phased array payload to increase the possibility of experiments which can be conducted during SKOUT's lifetime. For example, a secondary receive payload will be developed in-house to provide bi-directional links across the Space Research and FSS K-band (17.8-23.6 GHz). This receive system is envisioned to consist of a simple patch element, or possibly a small 4 element array. If an array design is implemented, an opportunity exists to test out a different silicon chipset to compare performance across RFIC manufacturers.

A number of experimental links are planned, as depicted in Fig. 2, to test the performance of 5G-based phased array technology, including high order modulations, high data rate demonstrations, multiple access relay capability, ground and space-based data downlinks, interoperability with NASA and commercial systems, as well as potentially advanced phased array-based sensing applications. Presently, NASA GRC is working with Cesium Astro for the development of the phased array and software defined radio (SDR) platforms and is planning to launch in the 2022 timeframe aboard a $6 \mathrm{U}$ CubeSat in low earth orbit.

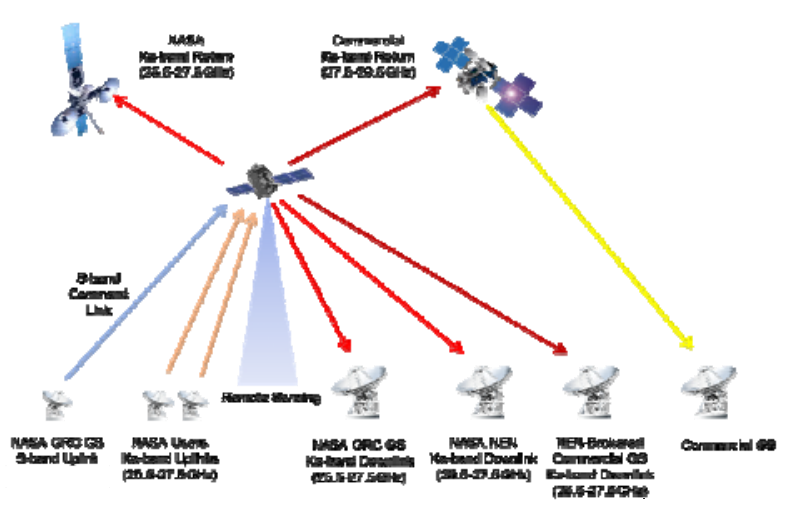

Fig. 2. Proposed experiments to be conducted during SKOUT mission life.

\section{B. Lunar Space Applications}

In the lunar regime, an architecture is being planned wherein a lunar gateway is placed in a near-rectilinear halo orbit that may serve as the primary data pipe back to earth, as depicted in Fig. 3. However, due to the large range of that orbit from the lunar south pole, additional smaller satellite lunar orbiters are being planned to provide higher rate links to surface assets on the ground (shown by the red orbits in Fig. 3). As depicted in Fig. 4, phased array technology could play a significant role in providing high data throughput links from the lunar surface to the lunar relay. This phased array technology may employ multi-beam technology or, at a minimum, fast steering capabilities, to support multiple users distributed across the lunar surface. In this way, higher gain beams will then be able to minimize the burden of lunar communications assets where power and pointing capability may be difficult. While phased arrays are being considered, at the time of this writing, the final lunar communications architecture is yet to be determined.

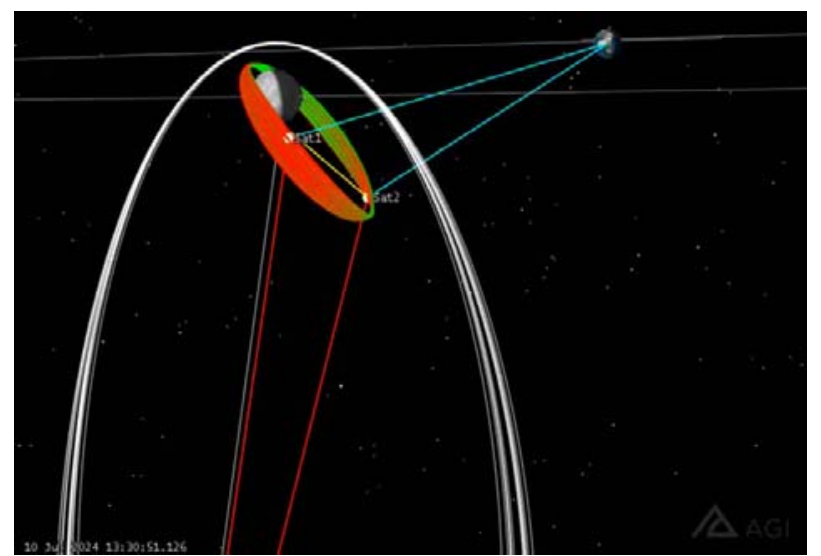

Fig. 3. Proposed lunar relay architecture with Gateway orbit (show in white) and a series of initial SmallSat lunar relays in a lower lunar orbit (shown in red), with associated links. 


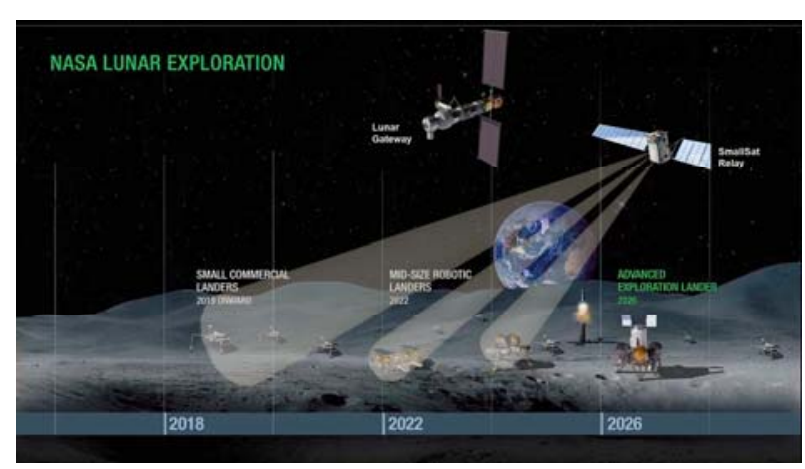

Fig. 4. Depiction of the use of multibeam phased array technologies to establish high rate links to lunar surface assets with minimal user burden. Modified from [9]

\section{Aeronautics Applications}

Within the framework of aeronautics applications, the growing demand for UAS operating at BLOS has resulted in increased interest in the use of commercial satellite communications capabilities for UAS command and control (C2) communications [10]. Recently, spectrum allocations were granted to aeronautics applications in the $\mathrm{Ku}$ and $\mathrm{Ka}$ FSS bands (12-18 GHz, 27.5-30 GHz) on a non-interference basis in order to improve the capacities of the future urban air mobility (UAM) market and provide potential BLOS links. Being implemented on a shared basis implies that strict spectrum transmission masks would need to be implemented in order to prevent interference with existing legacy systems operating in these bands. For example, the International Telecommunications Union (ITU) has released the mask requirements for $\mathrm{Ku}-\mathrm{band}$, as depicted in Fig. 5 [11].

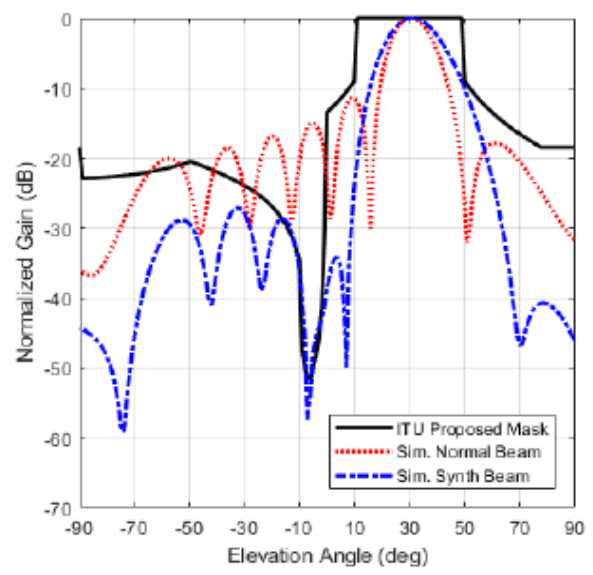

Fig. 5. Proposed ITU mask for UAS BLOS SATCOM at an altitude of 914 $\mathrm{m}$ and a $40^{\circ}$ latitude with simulated steered beam and optimized null for a 64-element phased array [12].

It thus becomes extremely evident that standard reflector-based antennas will not be able to meet the mask requirements identified by the ITU in the Ku-band, for example, necessitating the use of the flexible beamforming capabilities afforded by phased array technologies. Since
2017, NASA GRC has been investigating the use of siliconbased beamformer technologies to demonstrate conformal phased arrays for the UAS architecture for SATCOM BLOS links [11, 12]. Fig. 6 shows the prototype 64-element phased array developed in-house, which utilizes Anokiwave beamformer chipsets. Measurements in an antenna range were performed to validate null-steering performance of the array and demonstrate that the ITU mask requirements could be met with this technology and is shown in Fig. 7(a). In 2019, a follow-on flight demonstration was conducted at the NASA Armstrong Flight Research Center (AFRC) on board a T-34C aircraft to validate the performance of this phased array in a realistc environment.

Fig. 7(b) shows the performance of the array mounted to the T-34C aircraft and demonstrates that while the mounting conditions and aircraft geometry may not impact the main beam steering performance, it does have a dramatic effect on the null-steered beam. It should be noted that the original beam steering configuration of this array was optimized for a different platform (the Global Hawk UAS), but due to unforeseen issues, was last minute changed to the T-34C. This situation revealed yet another potential advantage of phased array technology ensuring that interference masks can be met irrespective of the platform it is operating on. As such, follow-on research at GRC for the use of phased array technologies in interference mitigation for the multitude of various potential UAS platforms will focus on the techniques and requirements for tunability of phased array technology to optimize performance based on the specific platform of operation.

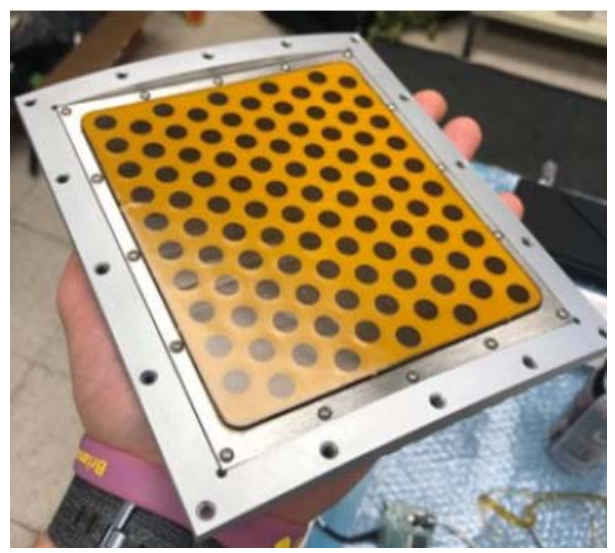

Fig. 6. Prototype conformal phased array antenna developed by NASA GRC utilizing the Anokiwave beamformer chipset [12]. 


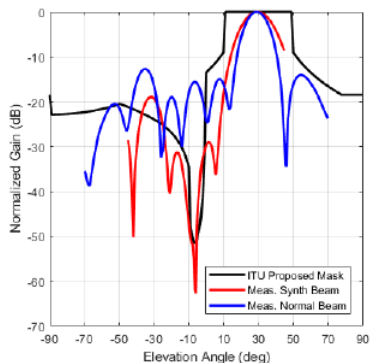

(a)

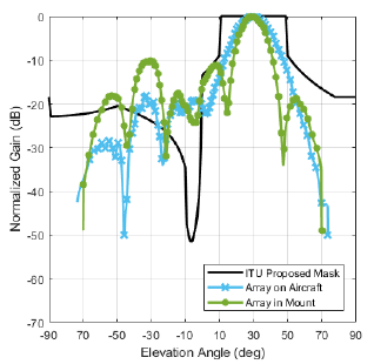

(b)
Fig. 7. (a) measured performance of the standard beam steering (blue) and null-beam steering (red) of the prototype 64-element array in the antenna

range, and (b) measured performance of the null-beam steered array

measured in the range on a representative mount (green) and in the hangar integrated on an aircraft (cyan) [12].

\section{CONCLUSIONS}

As future space and aeronautics architectures undergo radical architecture changes over the next decade, it is clear that phased array technologies will play a critical role. Several scenarios that NASA is presently considering for near earth, lunar space, and aeronautics applications that may only be realizable through the flexibility that phased array technology affords, were presented in this paper. Particularly, as space and aeronautics user platforms continue to shrink in size and available power and increase in demand for connectivity to many nodes, low cost, low SWaP phased array solutions become a must. To help advance this technology, NASA GRC has continued to research and invest in the exploitation of silicon-based ICs, particularly those being developed in the 5G band, due to the cross-over with NASA's Ka-band spectrum utilization.

\section{ACKNOWLEDGMENT}

The work presented in this paper has been supported by NASA's Human Exploration and Operations Mission Directorate (HEOMD) Space Communications and Navigation (SCaN) program, as well as NASA's Aeronautics Research Mission Directorate (ARMD) Convergent Aeronautics Solutions (CAS) program.

\section{REFERENCES}

[1] Mitsubishi Electric Corporation Public Relations Division, "Mitsubishi Electric's New Multibeam Multiplexing 5G Technology Achieves 20Gbps Throughput”, January 21, 2016

[2] A. M. Sayeed, "Millimeter-Wave MIMO Architectures for 5G Gigabits Wireless,” GlobeCom Workshop on Emerging Technologies for 5G Wireless Cellular Networks, December 8, 2014.

[3] 5G Americas White Paper, “Advanced Antenna Systems for 5G”, August 2019

[4] "5G New Radio Solutions for Chipset and Device Designers," Keysight technologies.

[5] "Bits to Beams: Chipset for 5G mmWave Radio," Microwave Journal, August 10, 2019.
[6] Agenda Item 10, United States Proposal for the Work of the Conference, World Radiocommunications Conference (WRC-19), October-November 2019

[7] M. Albulet, "SpaceX Non-Geostationary Satellite System”, FCC Application SATLOA2016111500118, November 2016

[8] M. Neri, “Telesat LEO: Fiber quality connectivity everywhere” ITU Regional Seminar for CIS and Europe, June 2018

[9] "Forward to the Moon: NASA's Strategic Plan for Lunar Exploration”, May 2019, NASA Public Release Document

[10] D. S. Ponchak, E. Auld, G. Church, and S. Henriksen "A Summary of Two recent Command and Control (C2) Communications Feasibility Study,” 2016 IEEE Aerospace Conference, Big Sky, MT.

[11] J. Downey, B. Schoenholz, M. Piasecki, R. Kerczewski, "Phased Array Antenna for the Mitigation of UAS Interference," 2018 Integrated Communications, Navigation, Surveillance Conference, April 2018.

[12] M. Piasecki, P. Slater, J. Downey, B. Schoenzholz, K. Lambert, "Active Array Measurements using the Portable Laser Guided Robotic Metrology System”, to be published.. 